\title{
Colonoscopic abnormalities in patients with IBD in a referral hospital in Salvador - Bahia
}

\begin{abstract}
Introduction: Inflammatory bowel disease (IBD) comprises a group of chronic and idiopathic diseases resulting from the inappropriate activation of the mucosal immune system. It has as main categories nosológical cateogories Crohn's disease $\mathrm{CD}$ and Ulcerative Colitis - UC. Colonoscopy is the exam of choice to diagnose and differentiate these two main forms of IBD involvement. It may be observed that UC involves a process of contiguous disease, which affects the rectum and progresses proximally, the intestine of segmental form, characterized by having healthy areas that separate inflamed areas.
\end{abstract}

Objective: To describe the changes observed in colonoscopies, of patients with indication and conclusion of Inflammatory Bowel Disease, performed at Hospital de Referênce hospital in Salvador.

Methods: Case series from January 2016 to May 2017 were selected for the study, all the colonosc reports with non- defined Intestinal Inflammatory Disease, CD and $\mathrm{UC}$, in the indication of the examination, at the conclusion (diagnosis) or both. The characteristics of the population and of the exams besides the colonoscopic alterations were described.

Results: A total of 130 colonoscopies were studied, of which 76 (58.5\%) were performed in female patients and $54(41.5 \%)$ in the male sex. The number of exams with diagnostic impression: normal examination, was only $22(16.9 \%)$. One hundred and eight $(83.1 \%)$ exams presented different alterations such as: edema, hyperemia, erosion, ulceration, enantema, polyps, pseudopolyps and alteration of the vascular pattern.

Conclusions: The study showed that changes observed in colonoscopies, especially inflammatory ones, such as: edema, hyperemia, enantema, vascular pattern alteration, erosion and ulceration, are frequently present in patients with Inflammatory Bowel Disease, both in the group that still had not defined its etiology when specific for CD and UC.

Keywords: Inflammatory bowel disease, colonoscopy, ulcerative colitis, crohn's disease, colonoscopic changes

\section{Introduction}

Inflammatory bowel disease (IBD) comprises a group of chronic and idiopathic diseases of poorly understood pathogenesis. ${ }^{1}$ It is also defined as a chronic condition resulting from the inappropriate activation of the mucosal immune system. ${ }^{2}$ This group has as main nosological categories, Crohn 's disease - $\mathrm{CD}$ and Ulcerative Colitis - UC. ${ }^{3}$ The etiology of IBD is unknown and has a multifactorial character. ${ }^{4}$

IBD can affect people of both sexes and in any age group, but it predominates between 20 and 40 years of age, impacting in an important way the quality of life, the social, labor and economic activity, of the individuals affected by it. ${ }^{5}$ The $\mathrm{CD} \& \mathrm{UC}$ are the two main clinical entities involved in IBD, but there is the Indeterminate Intestinal Inflammatory Disease (IIID) that cannot be classified as the diagnosis of exclusion. To differentiate the two major entities, UC is considered to involve a process of contiguous disease, which affects the rectum and progresses proximally, in addition to involving varying lengths of the colon. In contrast, CD usually involves the small intestine and large intestine in a segmental manner, characterized by having healthy areas that separate inflamed areas. ${ }^{6}$

$\mathrm{CD}$ is transmural in appearance, that is, it usually compromises the entire intestinal wall and can affect any part of the digestive tract,
Volume 10 Issue I - 2019

\author{
Taísa Maria Brito Amorim,' Lana Ferreira \\ Moreira,' Rafaela Mendonça Leal,' Adriana \\ Conceição de Mello Andrade, ${ }^{2}$ Carlos \\ Ramon Silveira Mendes ${ }^{3,4}$ \\ 'Acadêmica of Medicine, Faculty of Technology and Sciences, \\ Brazil \\ ${ }^{2}$ Professor at the Faculty of Technology and Sciences, Brazil \\ ${ }^{3}$ Preceptor Coloproctology Service of the General Hospital \\ Roberto Santos, Brazil \\ ${ }^{4}$ Coloproctology Chief of Hospital Santa Izabel, Brazil
}

Correspondence: Carlos Ramon Silveira Mendes, Rua Santa Izabela NI 00 Tower I fit 1002, CEP 40221225, Salvador Bahia Brazil,Tel +55 (7I) 999715852 ,

Email proctoramon@hotmail.com

Received: December 27, 2018 | Published: February 21, 2019 occurring more often in the distal small intestine, and occasionally in the right colon. ${ }^{6}$ Some of the morphological changes observed in Crohn's disease are: edema, reduction of mucus-producing cells, intestinal crypt hyperplasia, erosions, ulcerations, granulomas, lymphoid aggregates, dilation of lymphatic vessels in the tunica submucosa, and of the architecture of the submucosal plexus. ${ }^{7,8}$

The most characteristic histological manifestations, initially occurring in this disease, are: the formation of ulcers from the inflammatory process, which can lead to lumen narrowing, thickening of the intestinal wall and formation of granulomas in the mucosa. UC in divergence with $\mathrm{DC}$ is frequently restricted to the mucosa and has an upward involvement pattern, which initially involves a straight line and extends proximally throughout the colonic region. ${ }^{9}$ The degrees of involvement range from proctitis, when the disease is limited to the rectum, until pancolitis when it expands through the colon reaching the cecum. ${ }^{10}$ In it, ulceration often does not reach the muscle of the mucosa and inflammation is limited to the lamina propria. ${ }^{11}$ It is possible to observe in all forms of the disease the presence of polyps and pseudopolyps. ${ }^{12}$

The diagnosis of inflammatory bowel disease is performed by clinical data, radiological and histological findings in endoscopic biopsies and surgical resection pieces, when present. ${ }^{13}$ Of the different endoscopic methods, colonoscopy can be considered the 
main diagnostic tool in this group of diseases, since it is a widely used procedure for the detection and treatment of colonic disorders. ${ }^{14}$ It is, therefore, the first line examination for diagnosis and followup of patients with IBD, since the main differences between CD and UC can only be seen endoscopically and by anatomopathological examination. ${ }^{15}$

\section{Goals}

To determine the frequency of the main colonoscopic changes observed in colonoscopies of patients with indication and diagnosis of Inflammatory Bowel Disease.

\section{Methods}

A case series study was carried out, where 130 colonoscopies of patients of both sexes with indication and diagnosis of Inflammatory Bowel Disease were analyzed. Inclusion criteria: all colonoscopies of patients, performed from January 2016 to May 2017, attended by the referral hospital, who had as indication or diagnosis of their examination: Undetermined Inflammatory Bowel Disease, Crohn's Disease and Ulcerative Colitis. Exclusion criteria: colonoscopies without indication or diagnosis of IBD. The preparation of the colon is performed with $10 \%$ mannitol. The patient is instructed not to perform meal with residues the night before the examination. Patients undergo sedation at the discretion of the anesthesiologist. The colonoscopic examination was considered complete when it reached the cecum or terminal ileum. The work was submitted to the approval of the Research Ethics Committee (REC). The commitment of not identifying the patient was agreed by the researcher, with full confidentiality of the identity of the patients participating. The research did not offer disadvantages or damages to the patients involved in the study, since the type of analysis was done through the contact with colonoscopy reports, where there was no direct contact with them, so the application of the consent term was dispensable. The analyzed variables were: age, sex, origin, indication, examination condition, alterations and conclusion. As for the descriptive statistics, for absolute and absolute frequencies, the absolute and relative frequencies were used. The numerical variables were analyzed according to the mean and standard deviation.

\section{Results}

We analyzed 130 colonoscopies with indication and diagnosis of IBD. The mean age was 44.14 years with a standard deviation of 15.70 years for more or less. The female sex showed a higher incidence, representing $58.5 \%$ (76) of the total, and the male sex totaled $41.5 \%$ (54) of the patients. The lowest age was 9 years, and the maximum age was 89 years. Regarding the origin of the patient, $86.9 \%$ (113) were of external origin to the hospital, and only $13.1 \%$ (17) came from outpatient clinics or internal emergency. The exams were considered complete when performed to the cecum or terminal ileum, totaling $91.5 \%$ (119) of the exams. Only $8.5 \%$ (11) of them did not reach this location, due to intensely inflamed and friable mucosa with risk of perforation of intestinal loops or elevated lesion that impeded the progression of the endoscopic camera. The condition of the test was divided into good, fair, poor and bad, where $93.1 \%$ (121) had good conditions and only $6.9 \%$ (9) presented regular conditions of accomplishment. The number of normal exams was only $22(16.9 \%)$, and $108(83.1 \%)$ presented abnormalities in the normality pattern. Indications for performing colonoscopies are listed in Table 1, with UC being the most frequent indication in $41.5 \%$ (54) of the exams.
The most frequent colonoscopic changes are listed in Table 2, with the most frequent being in the $\mathrm{CD}$ group hyperemia and ulceration in $11.5 \%$ (15) of the patients, and in the group with UC, edema and erosion, in $18.5 \%$ (24). The least frequent alterations were the alteration of the vascular pattern that occurred in $0.8 \%$ (1) of patients with $\mathrm{CD}$, and the presence of polyps that occurred in $4.5 \%$ (6) of individuals with UC.The main findings of the analyzed studies are described in Table 3, with the most common being the UC diagnosis in $34.60 \%$ (45) and the less frequent presence of active IBD in 3\% (4) of the patients.

Table I Indication of the studied colonoscopy exams

\begin{tabular}{lllllll}
\hline & RCU & A.D & $\begin{array}{l}\text { IBD not } \\
\text { defined }\end{array}$ & Diarrhea & Bleeding & $\begin{array}{l}\text { Previous } \\
\text { surgery }\end{array}$ \\
\hline $\begin{array}{l}\text { Absolute } \\
\text { frequency }\end{array}$ & 54 & 41 & 16 & 12 & 6 & 1 \\
$\begin{array}{l}\text { Relative } \\
\text { frequency }\end{array}$ & $41.50 \%$ & $31.50 \%$ & $12.30 \%$ & $9.20 \%$ & $4.70 \%$ & $0.80 \%$
\end{tabular}

Source: Survey data (2017).

Table 2 Colonoscopic changes in patients with CD and UC

\begin{tabular}{lll}
\hline Change & Crohn's disease & Ulcerative colitis \\
Edema & $9(6.9 \%)$ & $24(18.5 \%)$ \\
hyperemia & $15(11.5 \%)$ & $15(11.5 \%)$ \\
soreness & $15(11.5 \%)$ & $11(8.4 \%)$ \\
Erosion & $8(6.15 \%)$ & $24(18.5 \%)$ \\
Change of Vascular Pattern & $1(0.8 \%)$ & $16(12.3 \%)$ \\
polyps & $6(4.5 \%)$ & $6(4.5 \%)$ \\
pseudopolyp & $7(5.4 \%)$ & $12(9.2 \%)$ \\
\hline
\end{tabular}

Source: Survey data (2017).

Table 3 Key findings of the studied colonoscopies

\begin{tabular}{lll}
\hline Conclusion & Absolute frequency & Relative frequency \\
\hline RCU & 45 & $34.60 \%$ \\
A.D & 23 & $17.70 \%$ \\
DII not def. & 10 & $7.70 \%$ \\
IBD activity & 4 & $3 \%$ \\
Others & 26 & $20 \%$ \\
Normal examination & 22 & $17 \%$ \\
\hline
\end{tabular}

Source: Research Data (2017).

\section{Discussion}

In the present study, $58.5 \%$ of the colonoscopic tests performed were female, and about $41.5 \%$ were male. The literature shows that Inflammatory Bowel Disease can occur in both sexes,${ }^{16}$ being slightly more frequent in the female, because the immunological component is involved in the pathology of this group of diseases. ${ }^{17}$

In addition, it was observed with this sample that, among the female population, there was a lower frequency of Crohn's Disease (16.1\%) than with Ulcerative Colostomy (29.2\%). This is not compatible with the literature, which shows the female sex as more frequent than the male in individuals with Crohn's disease. This may be linked to the fact that this study for having taken into account only one period of analysis, was not able to determine the same finding of the others. ${ }^{18}$

The mean age of patients who underwent colonoscopy was 44.14 years with a standard deviation of 15.7 years. Other studies that have evaluated in some way a population with IBD show that the mean age ranges from 38 to 42 years. In the state of Santa Catarina, a study with a little more than 100 individuals 
was conducted, which evaluated the epidemiological profile of patients with IBD, showing that the mean age was 39.56 , with a standard deviation of \pm 14.24 years. Another study conducted in Denmark, mean age in the same patient profile was 42 years. ${ }^{19,20}$ In this study, the lowest observed age was 9 years and the maximum of 89 , being seen in only 2 patients, this low frequency may be due to the study sample itself, however, it still reveals what the literature says, that the DII usually affect young adult individuals, and may present in a bimodal form, that is, they have two peaks of manifestation. The first peak is in the age group of 15 to 40 years and the second between $60-70$ years of age. ${ }^{21,22}$ More than $90 \%$ of the colonoscopies performed in the study reached the cecum or terminal ileum and had good preparation conditions, which demonstrates that there was adequate recourse for a correct and complete evaluation of the colon changes in its completeness, since the literature proves the importance of arriving at these sites, in order to directly visualize the entire colonic mucosa, confirming or excluding the diagnosis of IBD in the patient, through the examination. ${ }^{23}$ Because it is a reference center in IBD, and because of the intestinal preparation necessary for the procedure to be performed locally, there is the possibility of these factors, which is why there was a high frequency of exams with good and adequate visualization intestinal. ${ }^{24}$ It is also known that colonoscopic examination in certain situations raises concerns for its performance, potential risks of perforation and bleeding - depending on the clinical situation to which the patient is involved, and should therefore be well indicated and done by a trained professional. ${ }^{25}$

When evaluating the alterations in the examinations performed, it was found that more than $30 \%$ of them presented edema and hyperemia in the mucosa of the colon, which according to the literature, is a finding pertinent to the inflammatory process triggered by IBD ${ }^{26}$ In the present study, the presence of edema had a frequency of $6.9 \%$ in the population with $\mathrm{CD}$, and about $18.5 \%$ in patients with UC. In addition to edema having been more frequent in patients with UC, erosion, alteration of vascular pattern and presence of pseudopolypsis were more frequent within this group, in order to corroborate with the literature that these findings are in fact more frequent in the context of ulcerative colitis, and may help differentiate the etiology of IBD, although it is not unique to either condition. ${ }^{27}$ The higher frequency of these findings in the UC, corroborate with the studies involving IBD, since they show the existence of an important variety of definitions and endoscopic parameters considered indispensable for their evaluation, such as: friability, edema, enantema, loss of vascular pattern, granularity of mucosa, presence of erosions and ulcerations, essential parameters to classify the activity and severity of the UC. ${ }^{28}$

Through this study it can also be observed that about $8.4 \%$ of the patients with UC presented ulceration, and approximately $11.5 \%$ with DC presented this same finding. Some studies present in the current literature, which aimed to differentiate colonoscopic changes in $\mathrm{CD}$ and UC, reveals that the presence of ulcers is frequent in Crohn's disease, being used as a variable that also helps in the differentiation of IBD type. ${ }^{29}$ The common ulcers observed in CD are: acidoid, longitudinal or serpeginatic ulcers and / or in the ileo-terminal..$^{30}$

This study showed that only $3.85 \%$ of the patients had stenosis and that all those who had stenosis presented with Crohn's disease, thus evidenced similarly to the information present in the studies, which states that inflammatory and cicatricial stenosis is in fact more frequent in the $\mathrm{CD}$, than in the $\mathrm{UC}$, due to the transmural involvement of the first. It is important to remember that stenosis in the UC can occur, however, it is usually due to malignant neoplastic processes. ${ }^{31,32}$ In the present study, only stenosis appeared, but IBD may cause other complications. In UC, intestinal perforation, fulminant colitis, toxic megacolon, increased risk of colorectal cancer, in $\mathrm{CD}$, can be seen, intestinal obstruction, abscess, fistulae, fissures, malabsorption and malnutrition, biliary diarrhea, bacterial overgrowth in the small intestine. $^{33}$

The study, although containing the colonoscopic alterations of the studied population, does not describe the results of the biopsies performed, since it was not possible to have access in the Hospital, an examination that would help in a better description of these diseases, being considered a limitation for the study. In addition, because it is a sample that is not so numerous, the distribution of colonoscopic alterations in the $\mathrm{UC}$ and $\mathrm{CD}$, have not really been reliable in the presentation of these diseases.

\section{Conclusion}

Most of the colonoscopies performed had adequate intestinal preparation and were performed properly. In this study, there was no significant difference between the number of women and men submitted to colonoscopies, with a slightly higher number of female subjects. Inflammatory alterations, such as edema, hyperemia, enantema, altered vascular pattern, erosion and ulceration, appeared frequently in patients with IBD, both in the group that had not yet defined its etiology when specific for CD and UC.

Findings such as: erosion, edema and alteration of the vascular pattern were more frequent in the population with UC. The presence of ulcers was more frequent in patients with Crohn's disease, and pseudopolyps in patients with UC. Finally, complications such as stenosis were more frequent and present only in the Crohn's population. The study presents divergent information from the existing literature, such as the lower frequency of IBD in the female sex, which can be investigated in a deeper way through new analytical studies, in addition to the reduced number of complications question whether there is a better screening and follow-up of patients with IBD.

\section{Acknowledgments}

None.

\section{Conflicts of interest}

This study does not present conflicts of interest subsidy or financial support.

\section{References}

1. Inman R. Clinical manifestations and diagnosis of arthritis associated with inflammatory bowel disease and other gastrointestinal diseases. 2017.

2. Kumar V. Robbins \& Cotran: Pathology - pathological basis of diseases $9^{\text {th }}$ ed. Rio de Janeiro: Elsevier. 2015.

3. World Gastroenterology Organization practice guidelines. Inflammatory bowel disease: a global perspective. 2009.

4. Beyer. P1 treatment nutritional doctor to the lower gastrointestinal tract diseases. In: Mahan LK, Escott-Stump S. Krause: Food, Nutrition and Diet Therapy. $12^{\text {th }}$ ed. River Janeito: Elsevier, 2010. p. 689-695. 
5. Rubin E, Palazza JP. Inflammatory bowel disease. In: Rubin E, et al. Rubin pathology: clinicopathological bases of medicine. $4^{\text {th }}$ ed. Rio de Janeiro: Guanabara Koogan, 2006. p. 710, 725-730.

6. Cambui YRS, Natali MRM. Inflammatory Bowel Diseases: Narrative review of the literature. Rev Fac Ciênc Avg Sorocaba. 2015;17(3):116119.

7. Monahan FD. Bowel problems. In: Monahan FD, editor. Phipps MedicalSurgical Nursing: Perspectives of Health and Disease. $8^{\text {th }}$ ed. Loures: Lusodidacta, 2010. p. 1284-1291.

8. Peppercorn MA, Cheiftz AS. Definition, epidemiology, and risk factors in inflammatory bowel disease. 2016.

9. Maranhão DDA, Vieira A, Fields T. Features and differential diagnosis of inflammatory bowel diseases. Brazilian Journal of Medicine. $2015 ; 103(1)$

10. Lyra JR, Bonardi HF, Schiochet VJ. Importance of colonoscopy screening polyps and colorectal cancer in patients with rectal polyps. Brazilian magazine coloproctology. 2005;226-234.

11. Moreno BJLBG. IBD Challenges of diagnosis. Dissertation (Master of Medicine) - University of Beira Interior Health Sciences, Covilha. 2014

12. Zaltman C. Inflammatory bowel disease: the relevance of the disease in Brazil? 2018.

13. Moreira J. How to diagnose and treat disease inflammatory intestinal. São Paulo. 2014

14. Azevedo M,Carlos A, Milani LR, et al. How to Diagnose and Treat Inflammatory bowel disease. 2017.

15. Silva M. Biomarkers In Intestinal Inflammatory Disease. Thesis (MS) Medical School, University of Porto, Porto, 2013.

16. Tontini GE, Vecchi M1, Pastorelli L, et al. Differential diagnosis in inflammatory bowel disease colitis: State of the art and future perspectives. World J Gastroenterol. 2015;21(1):21-46.

17. Hermano ARL. Epidemiological Studies. 2018.

18. Pagano M, Gauvreau K. Principles of Biostatistics. São Paulo: A pionee Thomson Learning, 2004

19. World Gastroenterology Organization Practice Guidelines. Inflammatory bowel disease: a global perspective, 2015.
20. Cambui Y, Natali M. Inflammatory Bowel Disease: Narrative Literature Review. 2018.

21. Rose JR, Silva J, Rose M. Epidemiological profile of Patients with Inflammatory Bowel Disease. Arquivos Catarinenses de Medicina. 2018;43(2):53-58.

22. Halling ML, Kjeldsen J, Knudsen T, et al. Patients with inflammatory bowel disease have Increased risk of autoimmune and inflammatory diseases. World J Gastroenterol. 2017;23(33):6137-6146.

23. Arantes J. Epidemiology and clinical characteristics of patients with inflammatory bowel disease. J Coloproctol. (Rio J.) [online]. 2017;37(4):273-278.

24. Rex DK, Schoenfeld PS, Cohen J, et al. Quality indicators for colonoscopy. Gastrointestinal Endoscopy. 2015;81(1):31-53.

25. Salviano F, Burgos M, Santos E. Socioeconomic and nutritional profile of patients with inflammatory bowel disease at a university hospital. Arq Gastroenterol. 2007;44(2):99-106.

26. Passos MAT, Chaves FC, Chaves-Junior N. The importance of colonoscopy in inflammatory bowel diseases. Arq Bras Cir Dig. 2018;31(2):e1374

27. Flores C. Inflammatory Bowel Diseases: Endoscopic monitoring. 2008.

28. Crohn's \& Colitis Foundation. Intestinal Complications IBD. 2019.

29. Ng WK1, Wong $\mathrm{SH} 2, \mathrm{Ng} \mathrm{SC}$. Changing epidemiological trends of inflammatory bowel disease in Asia. Intest Res. 2016;14(2):111-19.

30. Ferraz FB. Overview of Inflammatory Bowel Disease: Immunity and Susceptibility to Crohn's Disease and Ulcerative Colitis. Journal of Health Sciences. 2015;19(5):139-143.

31. Moreno B. Inflammatory Bowel Disease: Diagnostic Challenges. Thesis (MS) - Medical School, University of Beira Interior, Covilhã, 2014.

32. Roy M. Endoscopic diagnosis of inflammatory bowel disease.

33. Tôrres S. Correlation between endoscopic findings and established treatment in patients with IBD. 33 2011. f. TCC (Graduation) - Medical School, Hospital Municipal Civil Servants, São Paulo, 2011 